\title{
DÉCOMPOSITION PAR DUALISATION D'UN PROBLÈME NON LINÉAIRE DE BASSIN SÉDIMENTAIRE
}

\author{
JONAS $\mathrm{KOKO}^{1}$
}

\begin{abstract}
We propose a Lagrange multiplier based decomposition method for a nonlinear sedimentary basin problem. Using an additional unkown, the sedimentary basin problem is restated as a linearly constrained minimization problem. The resulting Uzawa algorithm solves, at each iteration, one linear system with a constant (sparse) matrix. Numerical experiments on a model problem show that, for large scale problems, our method is more efficient than the classical Newton-Raphson method.

Résumé. Nous proposons une méthode de décomposition/coordination, avec coordination par multiplicateur de Lagrange, pour un problème non linéaire de bassin sédimentaire. A l'aide d'une inconnue additionnelle, nous reformulons le problème de bassin sédimentaire comme un problème de minimisation avec contraintes linéaires. L'algorithme d'Uzawa résultant résout, à chaque itération, un système linéaire dont la matrice (creuse) est constante. Des résultats numériques sur un problème modèle montrent que notre méthode est plus efficace que la méthode de Newton-Raphson, pour des problèmes de grande taille.
\end{abstract}

\section{INTRODUCTION}

Nous nous intéressons dans ce papier à la résolution par une méthode de décomposition par dualisation du problème non linéaire en $x \in \mathbb{R}^{n}$ suivant

$$
A x+G(x)=b,
$$

où

- A est une matrice carrée de taille $n$, symétrique définie positive;

- $G(x)=\left(c_{1} e^{\alpha x_{1}}, \ldots, c_{n} e^{\alpha x_{n}}\right)^{T}$ est un vecteur de $\mathbb{R}^{n}$ avec $\alpha>0$ et $c_{i}>0$, pour $i=1, \ldots, n$;

- $b \in \mathbb{R}^{n}$ un vecteur constant connu.

Le problème (1) est issu de la discrétisation, en temps et en espace, de l'équation simplifiée (voir par exemple $[10,12])$ qui modélise l'évolution d'un bassin sédimentaire

$$
\begin{aligned}
& \frac{\partial}{\partial t} e^{\alpha p}-K_{11} \partial_{1}^{2} p-K_{12} \partial_{12}^{2} p-K_{21} \partial_{1}^{2} p-K_{22} \partial_{2}^{2} p=f, \text { dans } \Omega \\
& p=p_{D}, \text { au bord de } \Omega
\end{aligned}
$$

Dans l'équation (2), $p=p(x, t)$ est la pression inconnue, $\alpha$ est un coefficient positif, $K=\left(K_{i j}(x, t)\right)$ est le tenseur de perméabilité du bassin sédimentaire et $f=f(x, t)$ les forces de volume qui s'exercent dans le bassin

\footnotetext{
1 LIMOS, Université Blaise Pascal, CNRS UMR 6158 ISIMA, Campus des Cézeaux, BP 10125, 63173 Aubière cedex, France; e-mail: koko@sp.isima.fr

(C) EDP Sciences, SMAI 2007
} 
sédimentaire. Les propriétés du tenseur $K$ déterminent celles de la matrice $A$. Dans notre cas, $K$ est donc un tenseur symétrique définit positif.

Un bassin sédimentaire est un milieu poreux hétérogène constitué de plusieurs couches stratifiées déposées au cours du temps. La modélisation d'un bassin sédimentaire a pour objectif la simulation numérique de son évolution en prenant en compte la compaction, la formation et la migration des hydrocarbures.

Comme la matrice $A$ est creuse, la résolution de l'équation (1) peut se faire par la méthode de NewtonRaphson. En partant d'un vecteur $x^{0}$ initial, on itère suivant le schéma

$$
\begin{aligned}
& \left(A+\nabla G\left(x^{k}\right)\right) s^{k}=b-A x^{k}-G\left(x^{k}\right), \\
& x^{k+1}=x^{k}+s^{k},
\end{aligned}
$$

où $\nabla G\left(x^{k}\right)=\alpha \operatorname{diag}\left(c_{1} e^{\alpha x_{1}}, \ldots, c_{n} e^{\alpha x_{n}}\right)$. La matrice $A+\nabla G\left(x^{k}\right)$ reste donc creuse au cours du processus itératif. Mais pour $n$ très grand, le coût de la factorisation peut devenir prohibitif. D'où la recherche de méthodes de résolution adaptées évitant la factorisation de matrices à chaque itération. Nous proposons une méthode de décomposition/coordination avec coordination par multiplicateur de Lagrange. C'est une méthode de dualisation (par introduction d'une variable auxiliaire) du type de celles proposées dans $[1,5,6]$. Dans notre méthode, on ne résout, à chaque itération, qu'un système linéaire ayant $A$ comme matrice. Par conséquent, on n'effectue qu'une seule factorisation de Cholesky à l'étape d'initialisation. Les autres résolutions se réduisent alors à des substitutions inverses et directes.

Zakarian et Glowinski proposent, dans [12], une méthode de décomposition de problème non linéaire de bassin sédimentaire de type moindres carrés avec contraintes de la forme (1). Comme les contraintes sont non linéaires le calcul du pas de déplacement, dans une méthode descente, est assez coûteux.

Le plan de l'article est le suivant. Au $\S 1$ nous reformulons le problème (1) comme un problème d'optimisation sans contrainte. La dualisation est réalisée au $\S 2$. L'algorithme de décomposition est présenté au $\S 3$ et les résultats numériques au $\S 4$.

\section{Problème D'optimisation}

Dans cette section, nous reformulons (1) comme un problème d'optimisation. Soit $q$ et $h$ les fonctions, de $\mathbb{R}^{n}$ dans $\mathbb{R}$, définies par

$$
\begin{aligned}
q(x) & =\frac{1}{2}(A x, x)-(b, x), \\
h(x) & =\frac{1}{\alpha} \sum_{i=1}^{n} c_{i} e^{\alpha x_{i}},
\end{aligned}
$$

où $(\cdot, \cdot)$ est le produit scalaire de $\mathbb{R}^{n}$. La fonction $q$ est quadratique, fortement convexe puisque $A$ est définie positive. La fonction $h$ est convexe car sa matrice Hessienne

$$
\nabla^{2} h(x)=\alpha\left(\begin{array}{ccc}
c_{1} e^{\alpha x_{1}} & & \\
0 & \ddots & 0 \\
& & c_{n} e^{\alpha x_{n}}
\end{array}\right)
$$

est définie positive. Posons

$$
\Phi(x)=q(x)+h(x) .
$$

La fonction $\Phi$ est fortement convexe $q$ est fortement convexe et $h$ est convexe. Comme l'équation (1) est équivalente à

$$
\nabla \Phi(x)=0,
$$


elle caractérise l'unique point stationnaire du problème de minimisation convexe

$$
\min _{x \in \mathbb{R}^{n}} \Phi(x) .
$$

Par la même occasion, on a montré que l'équation (1) a une solution unique.

\section{Dualisation}

Introduisons la fonction $F$, de $\mathbb{R}^{n} \times \mathbb{R}^{n}$ dans $\mathbb{R}$, définie par

$$
F(x, y)=q(x)+h(y), \quad \forall(x, y) \in \mathbb{R}^{n} \times \mathbb{R}^{n} .
$$

La fonction $F$ hérite des propriétés de $q$ et $h$, i.e. $F$ est une fonction strictement convexe. Posons $X=\{(x, y) \in$ $\left.\mathbb{R}^{n} \times \mathbb{R}^{n}: x-y=0\right\}$. Il est évident que le problème (1) est équivalent au problème d'optimisation avec contraintes

$$
\min _{(x, y) \in X} F(x, y) .
$$

Posons $C=\operatorname{diag}\left(c_{1}, \ldots, c_{n}\right)$. La matrice $C$ est définie positive. Nous pouvons donc équiper l'espace $\mathbb{R}^{n} \mathrm{du}$ produit scalaire

et de la norme associée

$$
(x, y)_{C}=(C x, y), \quad \forall x, y \in \mathbb{R}^{n}
$$

$$
\|x\|_{C}=(C x, x)^{1 / 2} .
$$

Associons au problème (8), le Lagrangien $\mathcal{L}$, définie sur $\mathbb{R}^{3 n}$ par

$$
\mathcal{L}(x, y ; \lambda)=F(x, y)+(C \lambda, x-y) .
$$

Comme $F$ est convexe et les contraintes linéaires, le Lagrangien $\mathcal{L}$ admet un point-selle $(x, y, \lambda)$, caractérisé par le problème min-max

$$
\max _{\lambda \in \mathbb{R}^{n}} \min _{(x, y) \in \mathbb{R}^{2 n}} \mathcal{L}(x, y ; \lambda)=\min _{(x, y) \in \mathbb{R}^{2 n}} \max _{\lambda \in \mathbb{R}^{n}} \mathcal{L}(x, y ; \lambda)
$$

ou, de manière équivalente, par les équations de point-selle

$$
\begin{aligned}
A x & =b-C \lambda, \\
e^{\alpha y_{i}} & =\lambda_{i}, \quad i=1,2, \ldots, n, \\
C(x-y) & =0 .
\end{aligned}
$$

Les équations (10) et (11) représentent la phase de minimisation, tandis que (12) représente la phase de maximisation. De l'équation (11) on déduit que les composantes du multiplicateur de Lagrange $\lambda$ doivent être strictement positives et

$$
y_{i}=\frac{1}{\alpha} \ln \left(\lambda_{i}\right), \quad i=1, \ldots, n .
$$

Notons qu'avec le produit scalaire "naturel" de $\mathbb{R}^{n}$, le résultat (13) serait

$$
y_{i}=\frac{1}{\alpha} \ln \left(\lambda_{i} / c_{i}\right), \quad i=1, \ldots, n .
$$

Le choix du produit scalaire (9) a donc eu pour effet de simplifier l'expression de $y$.

En substituant $y$ dans le Lagrangien $\mathcal{L}$ par (13), il vient que

$$
\mathcal{L}(x ; \lambda)=q(x)+(C \lambda, x)+\frac{1}{\alpha} \sum_{i=1}^{n} c_{i} \lambda_{i}\left(1-\ln \left(\lambda_{i}\right)\right) .
$$


Supposons maintenant que $x=x(\lambda)$ est solution de l'équation de point selle (10) pour $\lambda$ donné. Alors, en tenant compte de (10) dans le Lagrangien (14), on obtient

$$
\mathcal{L}(x(\lambda) ; \lambda)=-\frac{1}{2}(A x, x)+\frac{1}{\alpha} \sum_{i=1}^{n} c_{i} \lambda_{i}\left(1-\ln \left(\lambda_{i}\right)\right) .
$$

On définit alors la fonction duale

$$
F^{*}(\lambda)=\min _{x: A x=b-C \lambda} \mathcal{L}(x, \lambda)=\mathcal{L}(x(\lambda), \lambda)
$$

de sorte que le problème dual de (8) est

$$
\max _{\lambda \in \mathbb{R}^{n}} F^{*}(\lambda)
$$

Notre méthode de décomposition est basée sur le problème dual (15). C'est donc une méthode de type Uzawa.

\section{Algorithme De DÉCOMPOSItion}

Nous considérons les algorithmes d'Uzawa qui construisent une suite maximisante de $F^{*}$ de la forme

$$
\lambda^{k+1}=\lambda^{k}+t_{k} \mu^{k},
$$

où $\mu^{k}$ est une direction de montée de $F^{*}$ et $t_{k}>0$ le pas de déplacement.

Pour construire un algorithme de montée du problème (15), il nous faut des informations sur les dérivées de la fonction duale $F^{*}$. En réalité (15) est un problème de maximisation avec contrainte (i.e. l'équation de point selle (10)). D'après (10), l'application $\lambda \mapsto x(\lambda)$ est linéaire et

$$
x(\lambda+t \mu)=x(\lambda)+t z
$$

où $z$ est la solution du problème de sensibilité

$$
A z=-C \mu .
$$

En fait $z$ n'est rien d'autre que la dérivée de $x$ par rapport à $\lambda$. Pour une direction $\mu$ donnée, la dérivée directionnelle de $F^{*}$ est

$$
\frac{\partial}{\partial \lambda} F^{*}(\lambda) \cdot \mu=-(A z, x)-\frac{1}{\alpha} \sum_{i=1}^{n} c_{i} \mu_{i} \ln \left(\lambda_{i}\right)
$$

Sachant que $z$ est solution de (17), l'expression (18) devient

$$
\frac{\partial}{\partial \lambda} F^{*}(\lambda) \cdot \mu=(C \mu, x)-\frac{1}{\alpha} \sum_{i=1}^{n} c_{i} \mu_{i} \ln \left(\lambda_{i}\right) .
$$

En tenant compte du produit scalaire (9) qui équipe $\mathbb{R}^{n}$, on déduit alors que le gradient de $F^{*}$ a pour composantes

$$
\frac{\partial}{\partial \lambda_{i}} F^{*}(\lambda)=x_{i}-\frac{1}{\alpha} \ln \left(\lambda_{i}\right), \quad i=1, \ldots, n .
$$

Pour une direction de montée $\mu^{k}$ fixée, le pas de déplacement $t_{k}$ est le réel strictement positif qui maximise la fonction $\phi(t)=F^{*}\left(\lambda^{k}+t \mu^{k}\right)$. Comme $F^{*}$ est une fonction strictement concave, cela revient à résoudre dans $\mathbb{R}$ l'équation non linéaire

$$
\phi^{\prime}(t)=\frac{\partial}{\partial \lambda} F^{*}\left(\lambda^{k}+t \mu^{k}\right) \cdot \mu^{k}=\sum_{i=1}^{n} c_{i} \mu_{i}\left[x_{i}^{k}+t z_{i}^{k}-\frac{1}{\alpha} \ln \left(\lambda_{i}^{k}+t \mu_{i}^{k}\right)\right]=0 .
$$


Cette équation peut être résolue par les méthodes classiques de résolution d'équations dans $\mathbb{R}$ (Newton-Raphson, sécante, Brent,..., voir par exemple [3]). Notons que la résolution de (21) ne nécessite pas le calcul du gradient de $F^{*}$ comme le ferait une recherche linéaire classique.

Si $\mu^{k}=\nabla F^{*}\left(\lambda^{k}\right)$ dans (16), on obtient une méthode du gradient; très peu efficace car $F^{*}$ est fortement non linéaire. Dans l'hypothèse de grandes valeurs de $n$, la direction du gradient conjugué est celle qui offre le meilleur compromis coût / espace mémoire $[2,3,8,9]$. A chaque itération $k$, la direction du gradient conjugué (Fletcher-Reeves) est donnée par

$$
\begin{aligned}
\beta_{k} & =\frac{\left\|\nabla F^{*}\left(\lambda^{k}\right)\right\|_{C}^{2}}{\left\|\nabla F^{*}\left(\lambda^{k-1}\right)\right\|_{C}^{2}}, \\
\mu^{k} & =\nabla F^{*}\left(\lambda^{k}\right)+\beta_{k} \mu^{k-1} .
\end{aligned}
$$

Nous pouvons maintenant présenter notre algorithme de décomposition de type Uzawa/gradient conjugué de Fletcher-Reeves.

\section{Algorithme UCG}

- $k=0$ : Initialisation: $\lambda^{0}$ donné.

- Calculer $x^{0}$ solution de

$$
A x^{0}=b-C \lambda^{0}
$$

- Gradient initial:

$$
\gamma_{i}^{0}=x_{i}^{0}-\frac{1}{\alpha} \ln \left(\lambda_{i}^{0}\right), \quad i=1, \ldots, n
$$

- Direction de montée initiale:

$$
\mu^{0}=\gamma^{0}
$$

- $k \geq 0$ : Supposons $\lambda^{k}, \mu^{k}$ et $x^{k}$ connus.

- Calculer $z^{k}$ solution de

$$
A z^{k}=-C \mu^{k}
$$

- Calculer $t_{k}$ en résolvant (21)

- Mise à jour

$$
\begin{aligned}
& \lambda^{k+1}=\lambda^{k}+t_{k} \mu^{k} \\
& x^{k+1}=x^{k}+t_{k} z^{k}
\end{aligned}
$$

- Nouveau gradient

$$
\gamma_{i}^{k+1}=x_{i}^{k+1}-\frac{1}{\alpha} \ln \left(\lambda_{i}^{k+1}\right), \quad i=1, \ldots, n
$$

- Direction du gradient conjugué

$$
\begin{aligned}
& \beta_{k}=\frac{\left\|\gamma^{k+1}\right\|_{C}^{2}}{\left\|\gamma^{k}\right\|_{C}^{2}}, \\
& \mu^{k+1}=\gamma^{k+1}+\beta_{k} \mu^{k} .
\end{aligned}
$$

On arrête les itérations dès que

$$
\min \left\{\left\|\gamma^{k}\right\|_{C}, \frac{\left\|\gamma^{k}\right\|_{C}}{\left\|\gamma^{0}\right\|_{C}}\right\}<\varepsilon
$$


On remarque qu'on ne résout qu'un système linéaire (à matrice creuse) à chaque itération. De plus la matrice du système linéaire est constante durant les itérations. On effectue donc une factorisation de Cholesky, une fois pour toutes, dans l'étape d'initialisation. La résolution des systèmes linéaires durant les itérations se réduit alors à des substitutions directes et inverses. C'est là le principal avantage de l'algorithme UCG.

La mise à jour de la variable primale $x$ peut-être omise dans l'algorithme UCG. Dans ce cas, il suffit de résoudre à la fin des itérations l'équation de point-selle (10) avec le bon multiplicateur $\lambda$.

\section{RÉSUltats NumÉRiQUeS}

Nous présentons dans cette section quelques résultats numériques illustrant le comportement de notre algorithme de décomposition sur un problème modèle. L'algorithme UCG est comparé avec l'algorithme de Newton-Raphson (4)-(5). On arrête les itérations de Newton (4)-(5) dès que

$$
\frac{\left\|x^{k}-x^{k-1}\right\|}{\left\|x^{k}\right\|}<\varepsilon
$$

Dans les deux algorithmes, la tolérance est $\varepsilon=10^{-6}$. Les deux algorithmes ont été implémentés en Matlab et testés sur un PC équipé d'un processeur Pentium $4(3 \mathrm{GHz})$ sous Linux.

Le bassin sédimentaire modèle est $\Omega=(0,10) \times(0,10)$. Le coefficient $\alpha$ dans (1) est $\alpha=10^{-4}$. Le tenseur de perméabilité est donné par

$$
K(x, y)=\left(\begin{array}{cc}
y(10-y)+1 & 0 \\
0 & x(10-x)+1
\end{array}\right)
$$

Les forces extérieures s'exerçant sur $\Omega$ (i.e. le second membre dans (2)) sont

$$
f(x, y)=\exp (\alpha x y(x-10)(y-10))-2 y(y-10)(1-y(y-10))-2 x(x-10)(1-x(x-10)) .
$$

La matrice $A$ et le vecteur $c=\left(c_{1}, \ldots, c_{n}\right)^{T}$ sont obtenus par la méthode des éléments finis. La taille du problème $n$ correspond donc au nombre de nœuds du maillage de $\Omega$. Nous avons d'abord discrétisé le domaine $\Omega$ avec un maillage d'éléments finis de 177 nœuds (et 312 triangles). Ce maillage initial a été ensuite raffiné pour produire des maillages de $665,2577,10145,40257,160385$ et 217313 nœuds. A titre d'exemple, la figure 1 montre la densité de la matrice $A$ pour $n=10145$.

La table 1 montre les performances comparées, en nombre d'itérations, des algorithmes de Newton-Raphson et UCG. Nous pouvons remarquer que le nombre d'itérations reste relativement faible pour les deux algorithmes. La diminution du nombre d'itérations de l'algorithme UCG quand $n$ augmente peut s'expliquer par le fait que l'approximation par éléments finis est meilleure lorsque que $n$ augmente. 


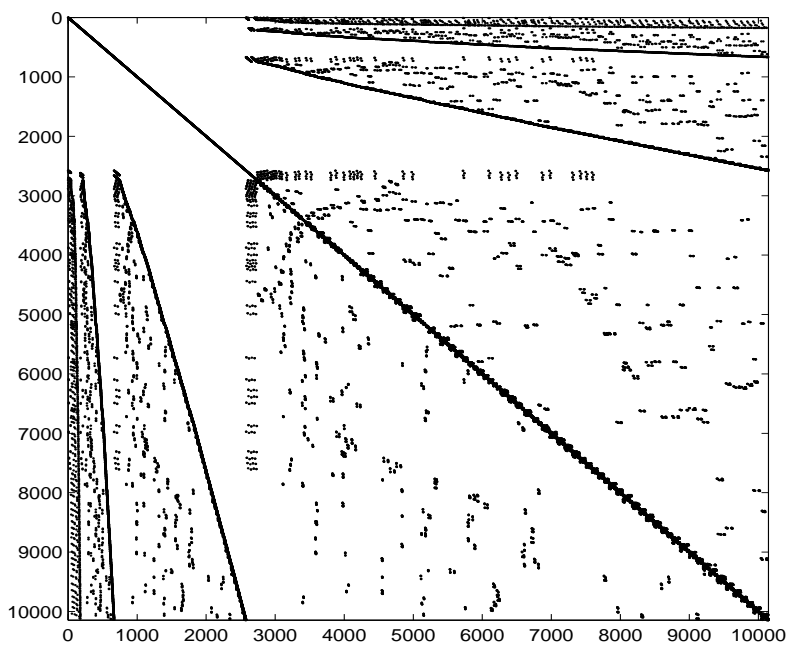

Figure 1. Densité de la matrice $A$ pour $n=10145$ : 70369 éléments non nuls sur $10145^{2}$

\begin{tabular}{ccc}
\hline & \multicolumn{2}{c}{ Nombre d'itérations } \\
$n$ & Newton-Raphson & Uzawa Gradient Conjugué \\
\hline 177 & 3 & 8 \\
665 & 3 & 7 \\
2577 & 3 & 6 \\
10145 & 3 & 5 \\
40257 & 3 & 5 \\
160385 & 3 & 4 \\
217313 & 3 & 4 \\
\hline
\end{tabular}

TABLE 1. Performances, en nombre d'itérations, des algorithmes de Newton-Raphson et Uzawa Gradient Conjugué

L'observation des temps CPU de la table 2 montre que notre algorithme est compétitif dès que $n>10000$. Pour l'algorithme UCG, nous avons distingué le cas où l'on prenais en compte le temps CPU de la factorisation (UCG I), du cas où ce temps était ignoré. Ce détail a de l'importance car l'équation (1) est résolue à chaque pas de temps lors de la simulation numérique de (2)-(3). Alors, si le tenseur de perméabilité $K$ ne dépend pas du temps, la matrice $A$ est constante. On effectue donc qu'une seule factorisation de Cholesky au début de la simulation. Dans ce cas le temps CPU de la factorisation peut être négligeable devant le temps de la simulation. Dans tous les cas, nous constatons que les gains en temps CPU obtenus avec notre algorithme sont de 28 à $55 \%$ (pour UCG I) et de 57 à $87 \%$ (pour UCG II), pour $n>10000$. 


\begin{tabular}{cccc}
\hline \multicolumn{3}{c}{ Temps CPU (en Sec.) } \\
$n$ & Newton-Raphson & algorithme UCG I & algorithme UCG II \\
\hline 177 & 0.01 & 0.05 & 0.05 \\
665 & 0.03 & 0.07 & 0.07 \\
2577 & 0.14 & 0.15 & 0.09 \\
10145 & 0.71 & 0.50 & 0.30 \\
40257 & 4.18 & 2.77 & 1.31 \\
160385 & 35.20 & 15.96 & 4.78 \\
217313 & 53.77 & 23.80 & 6.71 \\
\hline
\end{tabular}

TABle 2. Performances, en temps CPU, des algorithmes de Newton-Raphson et Uzawa Gradient Conjugué. Dans l'algorithme UCG II, le temps CPU de la factorisation de Cholesky n'est pas pris en compte.

\section{Conclusion}

Nous avons présenté une méthode de décomposition par dualisation d'un problème non linéaire de bassin sédimentaire. Les résultats numériques ont montré le bon comportement de notre méthode. Des études sont en cours pour améliorer l'algorithme présenté ici. La première piste est d'intégrer dans la décomposition le domaine pour avoir un algorithme de décomposition de domaine (voir par exemple $[4,11,12]$ ). Ensuite nous comptons prendre en compte la présence de faille dans le bassin sédimentaire.

\section{REFERENCES}

[1] Bresch D. and Koko J. An optimization-based domain decomposition method for nolinear wall laws in coupled systems. Math. Models Methods Appl. Sci., 14(7):1085-1101, 2004.

[2] Daniel J. The Approximate Minimization of Functionals. Prentice-Hall, Englewood Cliffs, NJ, 1970.

[3] Dennis J.E. and Schnabel R.B. Numerical Methods for Unconstrained Optimization and Nonlinear Equations. Classics in Applied Mathematics. SIAM, Philadelphia, 1996.

[4] Flauraud E., Nataf F., Faille I. and Masson R. Domain decomposition for an asymptotic geological fault modeling. C.R. Mecanique, 331:849-855, 2003.

[5] Glowinski R. and Le Tallec P. Augmented Lagrangian and Operator-splitting Methods in Nonlinear Mechanics. Studies in Applied Mathematics. SIAM, Philadelphia, 1989.

[6] Glowinski R. and Marocco A. Sur l'approximationpar éléments finis d'ordre un, et la résolution par pénalisation-dualité, d'une classe de problèmes de dirichlet non linéaires. RAIRO Anal. Num., 2:41-76, 1975.

[7] Jeannin L., Faille I. and GallouËt T. Comment modéliser les écoulements diphasiques compressibles sur des grilles hybrides. Oil and Gas Science and Technology - Rev. IFIP, 55(3):269-279, 2000.

[8] Luenberger D. Linear and Nonlinear Programming. Addison Wesley, Reading, MA, 1989.

[9] Minoux M. Programmation Mathématique: Théorie et Algorithmes I Dunod, Paris, 1983.

[10] Faille I. Modélisation bidimensionnelle de la génèse et de la migration des hydrocarbures dans un bassin sédimentaire. PhD Thesis, Université Joseph Fourier - Grenoble I, 1992.

[11] Willem F., Faille I., and Schneider F. Domain decomposition methods applied to sedimentary basin modeling. In BJøRSTAD, Espedal M.S., and Keyes D.E., editors, Ninth International Conference on Domain Decomposition Methods, pages 736-744. DDM.org, 1998.

[12] Zakarian E. and Glowinski R. Domain decomposition methods applied to sedimentary basin modeling. Math. Comput. Modelling, 30:153-178, 1999. 